\title{
Common Complications Post Tube Cystostomy Performed to Treat Obstructive Urolithiasis in Buffalo Calves
}

\author{
Akshay Kumar*, Azam Khan, CR. Swapnar, R. Sasikala, C. Sowbharenya, \\ Aswathy Gopinathan and Kiranjeet Singh
}

Division of Surgery, Indian Council of Agricultural Research-Indian Veterinary Research Institute, Izzatnagar, Bareilly, Uttar Pradesh, India

*Corresponding author

\section{A B S T R A C T}

Keywords

Urolithiasis, Post Tube Cystostomy, Buffalo Calves

Article Info

Accepted:

12 December 2020 Available Online: 10 January 2021
Urolithiasis refers to the formation of the stones in the urinary tract. Stones usually lodge in the lower portion of the urinary tract due to anatomical conformation. Tube cystostomy remains the method of choice with excellent results among other treatment protocols. Fifty cases of urolithiasis in male buffalo calves presented with complication post tube cystostomy were included to study the pattern of complications. Skin scalding and necrosis, obstruction of catheter, catheter dislodgement, and urine leakage and wound infection/dehiscence were the common complications observed post cystostomy in buffalo calves.

\section{Introduction}

Urolithiasis refers to the formation of the stones in the urinary tract. In animals specifically ruminants these stones usually lodge in the lower portion of the urinary tract due to anatomical conformation (Makhdoomi and Gazi, 2013). Both male and female affected with the urolithiasis, but urethral obstruction more commonly noted in male animals (Winter et al., 1987).Obstructive urolithiasis leads to very high mortaltity rate which is mainly due to rupture of urethra or urinary bladder (Gasthuys et al., 1993). There are several treatment protocol has been implicated for obstructive urolithiasis, but the tube cystostomy remain the method of choice with excellent results (Makhdoomi and Gazi, 2013).

The procedure is relatively simple, requiring a short duration of anaesthesia and resulting in restoration of full urethral patency in successful cases (Fortier et al., 2004). However, tube cystostomy is the preferred method but still this method having lots of complications if not adopted properly during surgery and postoperatively. Present study reports the major complications encountered after tube cystostomy in buffalo calves. 


\section{Materials and Methods}

Fifty buffalo calves presented to the Referral Veterinary Polyclinic, Indian Veterinary Research Institute, Izatnagar with postoperative complications of tube cystostomy were included in present study. All the animals were subjected to thorough anamnesis and clinical examination. Complications like urine leakage, wound infection and dehiscence, pus formation across the tract of the catheter, scalding and necrosis of skin, catheter dislodgment and obstruction of catheter were recorded.

\section{Results and Discussion}

All observed buffalo calves were male, with the age ranges from 2 to 6 months. Skin scalding and necrosis (21 cases, 42\%) (Fig.1), obstruction of catheter (12 cases, 24\%), catheter dislodgement (8 case, 16\%), urine leakage (5 cases, 10\%) and wound infection/ dehiscence (4cases, 8\%) (Fig.2) were the common complications recorded post cystostomy in buffalo calves.

Urine leakage is usually associated with the break in the continuity of the foley's catheter. After placement of defective catheter it results in dribbling of urine from the catheter, as a result the dribbled urine get accumulated in the dependent site (peritoneum, between the muscle layer and beneath the skin). Accumulated urine causes inflammation, fibrosis and necrosis of the site. Sometime urine gets entrapped in the muscle layers and subcutaneous tissue result in fibrosis of the tissue and the abdomen looked like pot bellied with hardness on the ventral aspect. Urine leakage can be prevented by evaluating the tube for any defect before using for the purpose of cystostomy.

Fig.1 Scalding and necrosis of skin observed in a calf

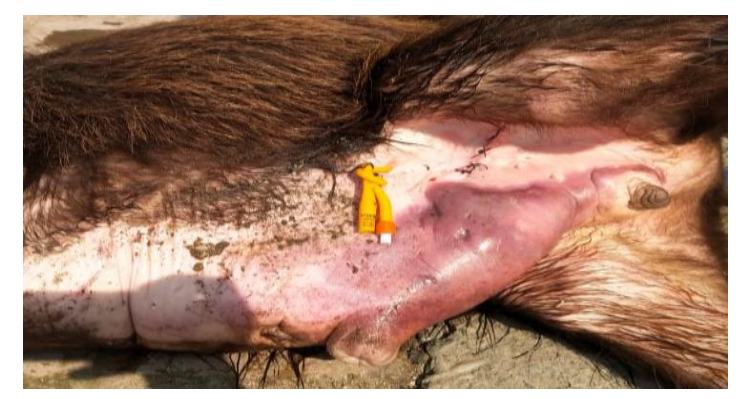

Fig.2 Post cystostomy urine seepage on the ventral aspect (A) and wound infection and dehiscence (B) in a calf

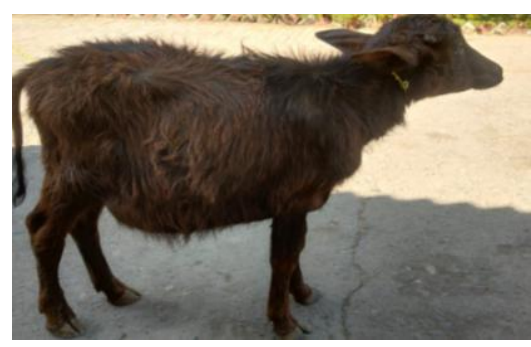

$\mathbf{A}$

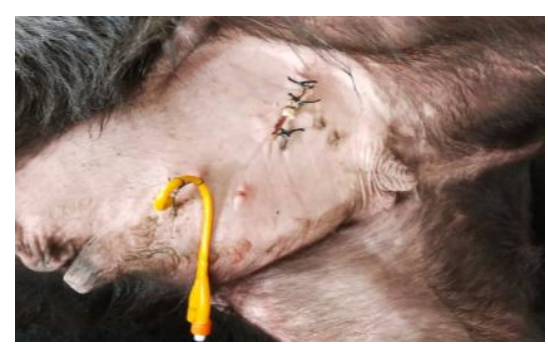

B 
Wound infection and dehiscence usually developed after using contaminated equipements during surgery and postoperative contamination of surgical wound with soils and dirt or any other contaminated foreign objects. The wound infection develops when wound is not cleaned properly with antiseptic solutions daily postoperatively. Once infection established in the wound it result in wound dehiscence with accumulation of pus around the suture line (Fig. 2). Wound infection and dehiscence can be prevented by adopting antiseptic dressing of the wound around the catheter and on the suture line with povidone iodine or chlorhexidine solution. Further antibiotics administered as adjunct therapy for prevention of infection. Antibiotic intended to use as prophylaxis reduce the incidence, but not effective for catheter induced infection, therefor CST of the catheter induced infectious material will help for selecting antibiotic and further treatment extended after removal catheter (Barsanti et al., 1987).

Tunnel created beneath the skin for securing the catheter usually results in pus formation across the catheter. This condition develops due to contaminated conditions adopted during tube cystostomy procedure. Sometimes the infected tract results in the ascending infection into the peritoneum and bladder that result in more serious complications. Catheter-induced infections are of particular concern with urolithiasis because cystitis, with sloughing of epithelial cells, may provide a nidus for further calculus formation.

Some time urethral rupture result in accumulation of urine around the ruptured urethra. Due to alkaline nature of the urine the skin scalding and necrosis starts around the urethra. Scalded skin looks pinkish blue. The scalding of skin progressively affects the other area and sometimes results in complete ventral abdomen scalding.
Catheter usually dislodged due to excessive filling of catheter balloon with water or normal saline that result in the rupture of balloon. After rupture catheter comes out of the bladder and dislodged from the bladder. Sometime animals pull catheter with the mouth that also result in dislodgment of catheter. High incidence of failure of the temporary cystostomy tube (Foley catheter) to remain fixed in position as a result of deflating of the balloon was the most serious complication encountered and required additional surgery (Rakestraw et al., 1995).

Calculi of ruminants are usually in the form of concrete that get stuck into the catheter and obstruct pathway for urine flow. Catheter also gets obstructive by blood and other fluid. During surgery thoroughly lavage urinary bladder to prevent future obstruction of urethra and catheter with calculi (Rakestraw et al., 1995).

Concentrate feed is usually rich in phosphorus and it is predisposing factor for calculi formation (Unmack, 2011). Re-obstruction of catheter can be prevented by recommending dietary management at the time of discharge, decrease the concentrate feed and protein rich feed in the diet so that antecedent factors for calculi formation is reduced.

In conclusions the complications are usually encountered due to improper surgical techniques, use of contaminated instruments for surgery, lack of proper postoperative care and mishandling of the cystotomy tube by client. Skin scalding and necrosis, obstruction of catheter and catheter dislodgement are common post tube cystostomy complications in buffalo calves

\section{References}

Barsanti, J.A., Blue, J. and Edmunds, J., 1985. Urinary tract infection due to 
indwelling bladder catheters in dogs and cats. Journal of the American Veterinary Medical Association, 187(4), pp.384-388.

Fortier, L.A., Gregg, A.J., Erb, H.N. and Fubini, S.L., 2004. Caprine obstructive urolithiasis: requirement for 2nd surgical intervention and mortality after percutaneous tube cystostomy, surgical tube cystostomy, or urinary bladder marsupialization. Veterinary Surgery, 33(6), pp.661-667.

Gasthuys, F., Steenhaut, M., De, A.M. and Sercu, K., 1993. Surgical treatment of urethral obstruction due to urolithiasis in male cattle: a review of 85 cases. The Veterinary Record, 133(21), pp.522-526.

Makhdoomi, D.M. and Gazi, M.A., 2013. Obstructive urolithiasis in ruminants-
A review. Veterinary World, 6(4). Rakestraw, P.C., Fubini, S.L., Gilbert, R.O. and Ward, J.O., 1995 . Tube cystostomy for treatment of obstructive urolithiasis in small ruminants. Veterinary Surgery, 24(6), pp.498-505.

Unmack, A.U.G.U.S.T.A., 1963. Constituents of calculi from the urinary tract of bulls and bullocks. Evidence of silica urolithiasis in cattle in Denmark. Constituents of calculi from the urinary tract of bulls and bullocks. Evidence of silica urolithiasis in cattle in Denmark.

Winter, R.B., Hawkins, L.L., Holterman, D.E. and Jones, S.G., 1987. Catheterization: an effective method of treating bovine urethral calculi. Veterinary medicine (USA).

\section{How to cite this article:}

Akshay Kumar, Azam Khan, CR. Swapnar, R. Sasikala, C. Sowbharenya, Aswathy Gopinathan and Kiranjeet Singh. 2021. Common Complications Post Tube Cystostomy Performed to Treat Obstructive Urolithiasis in Buffalo Calves. Int.J.Curr.Microbiol.App.Sci. 10(01): 839-842. doi: https://doi.org/10.20546/ijcmas.2021.1001.102 